\title{
Repositioning splint more effective than bite plane in the treatment of TMJ disk dislocation with reduction
}

Santacatterina A, Paoli M, Peretta R, Bambace A, Beltrame A. A comparison between horizontal splint and repositioning splint in the treatment of 'disc dislocation with reduction'. Literature meta-analysis. J Oral Rehab 1998 25; 8 1-88

Objectives To compare the effectiveness of repositioning splint and the bite plane in the treatment of disk dislocation with reduction (DDWR).

Data sources A Medline search 1985-1996.

Study selection Selection was based on description of the study, reliability of the data and homogeneity of sample employed. Six studies were included.

Data extraction and synthesis Percentage success for the treatment of pain and click and confidence intervals were calculated for each study.

\begin{tabular}{lcccccc} 
Results & \multicolumn{2}{l}{$\begin{array}{l}\text { Rescessful } \\
\text { Succestitioning Splint }\end{array}$} & \multicolumn{2}{c}{ Bite Plane } & ARR & NNT \\
treatment of & Mean & $95 \%$ Cl & Mean & $95 \%$ Cl & & \\
\hline Click & $74.5 \%$ & $69-80 \%$ & $17 \%$ & $7-27 \%$ & $52 \%$ & 2 \\
Pain & $79.8 \%$ & $72-87 \%$ & $33.3 \%$ & $18-49 \%$ & $46.5 \%$ & 2
\end{tabular}

Conclusions A correct treatment with the repositioning splint is the best procedure to follow in cases of DDWR. The systematic employment of the bite plane in treating DDWR is not supported by the necessary scientific evidence although this device has proved to be easier to use and more easily accepted by the patients.

Address for reprints: Dr Antonio Santacatterina, Via Baccarini No 9, 36015 Schio (Vicenza), Italy.

\section{Commentary}

This is a thoughtful and interesting article comparing the results of treatment by meta-analysis, of disc dislocation with reduction using two different methods.

The introduction gives a very clear summary of the theory behind the two different methods of treatment - the 'repositioners' and the 'functionalists'. The repositioners have the belief that every therapeutic effort should be made to restore the intra-articular anatomy. They quote the reasoning of Talents et al. CMD with articular origin is invariably progressive; the click, even without pain, is pathological; the disappearance of the click after therapy is always associated with the recapture of the disc; permanent rehabilitation must follow treatment. A detailed discussion with generous review of the literature follows, which refutes these conclusions and explains the rise of the 'selective repositioners'.

The 'functionalists' feel that the position of the disc is of little importance and they pay particular attention to neuromuscular and psychological factors. The disc position is immaterial; the click is often benign; therapy should concentrate only on creating the best conditions for the patient to recover; extensive rehabilitation or surgery should be reserved for those totally refractory to any other treatment. The authors quote Green and Laskin.

In the discussion which followed, the authors made the following points:

- The sample of articles was small, and this was due to the surprising inadequacy of essential data in the results of many studies.

- There were no accepted unequivocal criteria for the success of therapy for CMD.

- The conclusions could be made only for temporary occlusal therapy by means of splints.

- No information could be found for definitive longer-term therapy.

- There is no scientific evidence supporting the use of the bite plane in disc dislocation with reduction.

This was a very thorough paper and highlights the inadequacy of the results of many 'trials' of therapy to cure TMJ pain and dysfunction. The criteria for a successful treatment even, are not agreed. Also, this paper only considers patients who have a painful TMJ click (one of the papers considered all clicks, whether painful or not) and the total number of patients included for metaanalysis for both groups was small (bite plane for click 54, and pain 36; repositioning splint for click 212, and pain 114). The conclusions may be valid but should be interpreted with care.

It has to be recognised that most patients have pain additional to that emanating from the joint, and therefore no conclusions should be drawn regarding the treatment of pain felt in the side of the face or headache. It is these pains which are of far greater significance in the management of patients with CMD.

Limited though the conclusions from this paper may be, the paper itself serves to highlight the scientific inadequacy of many papers purporting to indicate successful therapy for pain associated with the TMJ. It concluded that further research is required, extending over a longer period, and using correct initial definition of method, before any recommendations for definitive treatment can be made.

\section{Richard Juniper \\ University of Oxford, Oxford, UK}

